\title{
ON KÖTHE SPACES
}

\author{
ROBERT WELLAND(1)
}

1. Köthe and Toeplitz in [7] and later Köthe in [8] and [9] began the study of certain pairs of subspaces of the space of all real sequences. Their theory has been generalized by Dieudonné [4], M. J. Cooper [3], and Lorentz and Wertheim [11]. Dieudonné, in his work, considers pairs $\left(\Lambda, \Lambda^{*}\right)$ of subspaces of the space of locally integrable functions on a locally compact $\sigma$-compact Hausdorff space $E$ with a Radon measure $\mu$. The spaces $\left(\Lambda, \Lambda^{*}\right)$, which are locally convex topologically complete vector lattices, are called Köthe spaces; well-known examples of these are the pairs $\left(L_{p}, L_{q}\right)$. We present two results which contribute to the theory as developed by Dieudonné; however, since the topology of the underlying topological space $E$ plays no role in these results, they are presented in a purely measure theoretic setting. This necessitates presenting some of the general theory of Köthe spaces.

The first of the two results generalizes the classical theorem: if $L^{r} \subset L^{s}$ and $r \neq s$, then $L^{r}$ is of the first category in $L^{s}$; the second result proves the equivalence of four statements; one of which is: $\Lambda^{*}$ can be identified with the topological dual of $\Lambda$.

2. This section contains definitions and facts from the general theory.

Let $(E, \mathscr{S}, \mu)$ be a separable totally $\sigma$-finite measure space [6]; here $E$ is a point set, $\mathscr{S}$ is a $\sigma$-algebra of subsets of $E$ and $\mu$ is a totally $\sigma$-finite measure. By a cover of $E$, we mean a subset $\mathscr{C}$ of $\mathscr{S}$ which satisfies:

(1) $\mathscr{C}$ contains a countable subset whose union is $E$,

(2) $\mu(C)<\infty$ for every $C$ in $\mathscr{C}$.

A real-valued function $f$ defined on $E$ is said to be $\mathscr{C}$-locally integrable if $\chi(C) f \in L^{1}(E, \mu)=L^{1}$ for every $C$ in $\mathscr{C}(\chi(C)$ denotes the characteristic function of $C)$. The set of such functions will be denoted $\Omega_{\mathscr{\ell}}$. In this space two functions are regarded as equivalent if they differ only on a set of measure zero. This simple notion of equivalence is not suitable if $E$ is not a measurable set; for then it is possible for a function to be locally integrable without being measurable.

The space $\Omega_{\varnothing}$ is a vector lattice where $f \vee g(x)=\max (f(x), g(x))$ and $f \wedge g(x)=\min (f(x), g(x))$. In addition to this, $\Omega_{\mathscr{C}}$ has a natural topology given by the seminorms

Received by the editors April 1, 1963.

(1) This work was sponsored by the National Science Foundation, Grant 19135. 


$$
N_{c}(f)=\int_{C}|f| d \mu, \quad C \in \mathscr{C} .
$$

The space $\Omega_{\mathscr{C}}$ is complete in two senses; first, it is topologically complete and second, it is a conditionally complete lattice. Recall, a lattice is said to be conditionally complete if each nonvoid subset with an upper bound has a least upper bound.

To show the topological completeness of $\Omega_{\mathscr{C}}$, let $\left(f_{\alpha}\right)$ be a Cauchy net. For any $C$ in $\mathscr{C}$ set

$$
L_{C}^{1}=\left(f \in L^{1}: \chi(C) f=f\right)
$$

and give it the norm topology. Consider $\left(\chi(C) f_{\alpha}\right)$; this is a Cauchy net in $L_{c}^{1}$. Because this space is topologically complete, there exists a limit $f_{C}$ in $L_{C}^{1}$ of this net. If $C_{1}$ and $C_{2}$ are two elements of $\mathscr{C}$, it is easy to show that $\chi\left(C_{2}\right) f_{C_{1}}$ $=\chi\left(C_{1}\right) f_{C_{2}}$ a.e. Thus, because $E$ is a measurable set, there exists a measurable function $f$ such that $\chi(C) f=f_{C}$ for every $C$ in $\mathscr{C}$. It is easily seen that $f \in \Omega_{\mathscr{C}}$ and that it is the limit of the net $\left(f_{\alpha}\right)$.

It follows that the space $\Omega_{\mathscr{C}}$ is conditionally complete because this is the case for each of the spaces $L_{C}^{1}$ and because $|g| \leqq|f|, f \in \Omega_{\mathscr{C}}$ implies $g \in \Omega_{\mathscr{C}}$.

Without changing the space $\Omega_{\mathscr{C}}$ or its topology, we could close $\mathscr{C}$ with respect to taking finite unions. Accordingly, we assume that $\mathscr{C}$ has this additional property. With this, we can find in $\mathscr{C}$ a sequence of sets $E_{1} \subset E_{2} \subset \cdots$ such that $E=\bigcup_{i=1}^{\infty} E_{i}$. From now on, we assume that $\mathscr{C}$, together with such a sequence of sets, is fixed.

It should be noted that although $\Omega_{\mathscr{C}}$ itself is not necessarily metrizable, it is contained in an $\Omega_{\mathscr{C}}$, which is. This is clear since $\mathscr{C}^{\prime}=\left(E_{i}\right)$ is a cover of $E$ and $\Omega_{\mathscr{C}} \subset \Omega_{\mathscr{G}}$. To see that $\Omega_{\mathscr{C}}$ is not necessarily metrizable, let $E=[0,1]$ and $\mu$ be Lebesgue measure. Set $I_{n}=[1 / n, 1 / n+1]$; and choose a set $\mathfrak{A}$ of $c$-subsets of the positive integers such that

(1) $\mathfrak{A}$ is closed with respect to finite unions,

(2) $\mathfrak{A}$ does not contain a countable set $\alpha_{1}, \alpha_{2}, \cdots$ such that $\alpha$ in $\mathfrak{U}$ implies $\alpha \subset \alpha_{i}$ for some $i=1,2, \cdots$, and

(3) $\bigcup(\alpha: \alpha \in \mathfrak{A})$ is the set of positive integers.

The existence of a set such as $\mathfrak{A}$ can be shown with only a modest amount of aggravation. The space $\Omega_{\mathscr{C}}$ has the desired property if $C=\left(C: C=\bigcup_{n \in \alpha} I_{n}\right.$ for some $\alpha$ in $\mathfrak{A}$ ); for $\mu(E)<\infty$ and (3) imply that $\mathscr{C}$ is a cover of $E$ while (1) and (2) imply that $\left(N_{C}: C \in \mathscr{C}\right)$ determines a nonmetrizable topology, i.e., it requires uncountably many seminorms to determine the topology.

3. Since the cover of $E$ is fixed for the rest of this work, we write $\Omega$ in place of $\Omega_{\mathscr{\zeta}}$. As in Dieudonné [4], the Köthe spaces are defined as follows: Let $\Gamma$ be any subset of $\Omega$ and set

$$
\Lambda=\Lambda(\Gamma)=\left(f \in \Omega: f g \in L^{1} \text { for all } g \in \Gamma\right)
$$


and $\Lambda^{*}=\Lambda(\Lambda)$. The following are some facts that follow readily from the definition of $\Lambda$ and the properties of $\Omega$ :

(a) $\Lambda\left(\Lambda^{*}\right)=\Lambda$.

(b) $\Lambda$ is a vector space.

(c) If $f \in \Lambda$ and $|g| \leqq|f|, g \in \Omega$, then $g \in \Lambda$.

(d) $\Lambda$ is a conditionally complete lattice.

(e) $\Lambda$ and $\Lambda^{*}$ are put in weak duality by the bilinear form $\int f g d \mu$.

This bilinear form is used to give $\Lambda$ several uniformities. Let $W$ be any set of weakly bounded normal subsets of $\Lambda^{*}$ whose union is $\Lambda^{*}$ (a set $B \subset \Lambda^{*}$ is said to be normal if $|h| \leqq|g|, g \in B$ and $h \in \Omega$ imply $h \in B$ ). Define seminorms $S_{B}$ by

$$
S_{B}(f)=\sup _{g \in B} \int f g d \mu,
$$

and denote the uniformity they define by $K_{W}$.

It can be shown, as in [4], that $\Lambda$ with the uniformity determined by these seminorms is not only a locally convex space but that it is topologically complete. Since $S_{B}\left(f_{1}\right) \leqq S_{B}\left(f_{2}\right)$, whenever $\left|f_{1}\right| \leqq\left|f_{2}\right|, \Lambda$ can be regarded also as a topological vector lattice.

The following three lemmas are needed in the work to follow.

Lemma 1. If $C \in \mathscr{C}$, then the mapping $f \rightarrow \chi(C) f$ of $\Lambda$ into $L_{C}^{1}$ is continuous. Denote the image of this mapping by $\Lambda_{C}$.

Proof. Let

$$
o_{C}=\left(f \in L_{C}^{1}: \int|f| d \mu \leqq 1\right) .
$$

It suffices to show that $\left(f \in \Lambda: \chi(C) f \in O_{C}\right)$ is a neighborhood of zero in $\Lambda$. Because $\mu(C)<\infty$, we have $\chi(C) \in \Lambda^{*}$. The sets in $W$ are normal and have $\Lambda^{*}$ as their union; therefore, there exists a $B \in W$ such that $\chi(C) \in B$ and consequently, $\left(g \in \Lambda^{*}:|g| \leqq \chi(C)\right) \subset B$. This last inclusion implies that

$$
\left(f \in \Lambda: S_{B}(f) \leqq 1\right) \subset\left(f \in \Lambda: \chi(C) f \in O_{C}\right)
$$

which in turn completes the proof.

If $S_{n}$ is a sequence of numbers with $S_{n+1} \leqq S_{n}$ and $\lim _{n \rightarrow \infty} S_{n}=0$, we write $S_{n} \downarrow 0$; if $S_{n+1} \geqq S_{n} \geqq 0$, we write $S_{n} \uparrow$. Similarly, if $f_{n}$ is a sequence of functions such that $f_{n}(x) \downarrow 0$ a.e. or $f_{n}(x) \uparrow$ a.e., we write $f_{n} \downarrow 0$ or $f_{n} \uparrow$.

LEMMA 2. Let $\left(f_{n}\right), f_{n} \geqq 0$, be a sequence in $\Lambda$ such that $f_{n} \uparrow ;$ then $\lim _{n \rightarrow \infty} S_{B}\left(f_{n}\right)$ $=S_{B}\left(\lim _{n \rightarrow \infty} f_{n}\right)$.

Proof. The lemma follows directly from two facts; first, if $g \in B$ then $|g| \in B$ and second

$$
\lim _{n \rightarrow \infty} \int f|g| d \mu=\int \lim _{n \rightarrow \infty} f_{n}|g| d \mu \text {. }
$$


Corollary. If $\left(f_{n}\right), f_{n} \geqq 0$, is a sequence in $\Lambda$ such that $f_{n} \uparrow$ and if for each $B \in W$ there exists a constant $M=M(B)$ such that $S_{B}\left(f_{n}\right)<M<\infty$ for all $n$, then $\Lambda$ contains an element $f$ such that $f_{n} \leqq$ for all $n$.

Proof. This corollary follows directly from Fatou's lemma.

Both Lemma 2 and its corollary are valid if the sequences $\left(f_{n}\right)$ are replaced by increasing nets. As a consequence of this, these results can be used in conjunction with a deep theorem of Nakano to give an alternate proof of the topological completeness of $\left(\Lambda, K_{W}\right)$. For an expository discussion of this point see Goffman [5].

Let $K$ be the uniformity on $\Lambda$ obtained when $W$ contains all weakly bounded normal subsets of $\Lambda^{*}$. We say $\Lambda^{*}$ can be identified with the dual of $(\Lambda, K)$ if for each continuous linear form $G$ on $(\Lambda, K)$ there exists a $g$ in $\Lambda^{*}$ such that

$$
G(f)=\int f g d \mu
$$

whenever $f \in \Lambda$. Note, each $g$ in $\Lambda^{*}$ defines a continuous linear form.

LEMmA 3. If $\Lambda^{*}$ can be identified with the dual of $(\Lambda, K)$, then $(\Lambda, K)$ is a tonnelé space (recall, a space is said to be tonnele if every convex closed balanced absorbing set is a neighborhood of 0 ; see [1]).

Proof. Let $U$ be a convex closed balanced absorbing set in $(\Lambda, K)$. We must produce a normal weakly bounded subset $B$ of $\Lambda^{*}$ such that

$$
B^{0}=\left(f \in \Lambda:\left|\int f g d \mu\right| \leqq 1 \text { for all } g \in B\right) \subset U .
$$

To do this, it suffices to show that $U$ contains a closed convex balanced absorbing normal subset $A$; for, if this can be done, then

$$
B=\left(g \in \Lambda^{*}:\left|\int f g d \mu\right| \leqq 1 \text { for } f \in A\right)
$$

will be a weakly bounded normal subset of $\Lambda^{*}$ and $B^{0}$ will be the weak convex closure of $A\left[1\right.$, p. 67]; the assumption that $\Lambda^{*}$ can be identified with the dual of $(\Lambda, K)$ implies that the weakly closed convex sets are the same as the $K$-closed convex sets $[1$, p. 67$]$; this in turn will imply that $A=B^{0}$, completing the proof. Let $f$ be any element of $\Lambda$. Towards showing the existence of a set such as $A$, we show there exists a positive number $\alpha$ such that

$$
I_{\alpha|f|}=(h \in \Lambda:|h| \leqq \alpha|f|) \subset U .
$$

Let $V$ be the subspace of $\Lambda$ of functions $h$ such that $|h| \leqq \beta|f|$ for some number $\beta$. If $E_{0}$ is the support of $f$, then the linear mapping $T$, defined by $T(h)(x)=h(x) /|f|(x)$ if $x \in E_{0}$ and $T(h)(x)=0$ otherwise, maps $V$ one-to-one and onto the space of essentially:bounded measurablerfunctions whose supports are contained in $E_{0}$. 
Let $L^{\infty}\left(E_{0}\right)$ denote the range of $T$. The space $L^{\infty}\left(E_{0}\right)$ with the essential supremum norm is a Banach space; hence it is tonnelé $[1$, p. 2].

If $V$ is given the induced topology, then the linear mapping $T$ is closed in addition to being an algebraic isomorphism. To see this, let $\left(k_{n}\right)$ be a sequence in $L^{\infty}\left(E_{0}\right)$ which converges to $k$, let $B$ be any weakly bounded normal subset of $\Lambda^{*}$ and notice that

$$
\sup _{g \in B} \int|f|\left(k-k_{n}\right) g d \mu \leqq\left\|k-k_{n}\right\|_{\infty} \sup _{g \in B} \int|f| g d \mu .
$$

The set $U_{0}=(h \in \Lambda: h \in \Lambda \cap U)$ is closed in $V$; it is also convex, balanced and absorbing in $V$. Thus, $T\left(U_{0}\right)$ has these properties in $L^{\infty}\left(E_{0}\right)$ and therefore, it is a neighborhood of 0 there, that is, there exists $\alpha>0$ such that $k$ in $L^{\infty}\left(E_{0}\right)$ and $|k|(x) \leqq \alpha$ a.e. implies $k \in T\left(U_{0}\right)$. This implies that $I_{\alpha|f|} \subset U_{0} \subset U$. For $f$ in $U$ choose any $\alpha=\alpha(f)$ such that $I_{\alpha|f|} \subset U$. Let $A^{\prime}=\bigcup_{f \in U} I_{\alpha|f|}$. Let $A$ be the smallest closed convex balanced subset of $\Lambda$ which contains $A^{\prime}$. This set is contained in $U$.

We need only show that $A$ is normal in order to complete the proof.

Let $A^{\prime \prime}$ be the smallest convex subset of $\Lambda$ which contains $A^{\prime}$. This set is normal and therefore it is also balanced. To see that $A^{\prime \prime}$ is normal, let $f$ be any element of $A^{\prime \prime}$ and suppose $h \in \Lambda$ such that $|h| \leqq|f|$. There exist functions $\bar{g}_{i}$ each from a set $I_{\alpha\left|f_{i}\right|}$ and positive constants $a_{i}, i=1, \cdots, n$, such that

$$
|f|=\sum_{i=1}^{n} a_{i} \bar{g}_{i}
$$

and $\sum_{i=1}^{n} a_{i}=1$. If $h(x)=0$ set $g_{i}(x)=0$ and if $h(x) \neq 0$ set $g_{i}(x)=h(x) \bar{g}_{i}(x) /|f|(x)$. The relationship $\left|g_{i}\right| \leqq\left|\bar{g}_{i}\right|$ implies $g_{i} \in I_{\alpha\left|f_{i}\right|}$. This in turn implies that $h=\sum_{i=1}^{n} a_{i} g_{i} \in A^{\prime \prime}$. The closure of $A^{\prime \prime}$ is convex and balanced; we now show that it is also normal.

Let $h$ be an element of $\Lambda$ which for some $f$ in the closure $\bar{A}^{\prime \prime}$ of $A^{\prime \prime}$ satisfies $|h| \leqq|f|$. Let $\left(f_{\gamma}\right)$ be a net in $A^{\prime \prime}$ which converges to $f$. The elements $\left|f_{\gamma}\right|$ are in $A^{\prime \prime}$; so that the elements

$$
h_{\gamma}=\left(f \wedge\left|f_{\gamma}\right| \wedge|h|\right) \operatorname{sign} h
$$

are also in $A^{\prime \prime}$. We show that $\left|h-h_{\gamma}\right| \leqq\left|f-f_{\gamma}\right|$ which implies $h \in A^{\prime \prime}$. Let $x$ be such that $h(x) \geqq 0$. If $\left|f_{\gamma}(x)\right| \geqq h(x)$, then $h_{\gamma}(x)=h(x)$. If $\left|f_{\gamma}(x)\right| \leqq h(x)$, then $h_{\gamma}(x)=\left|f_{\gamma}(x)\right|$ so that $\left|h(x)-h_{\gamma}(x)\right| \leqq\left\|f(x)|-| f_{\gamma}(x)\right\| \leqq\left|f(x)-f_{\gamma}(x)\right|$. A similar analysis shows $\left|h-h_{\gamma}\right| \leqq\left|f-f_{\gamma}\right|$ in the case that $h(x) \leqq 0$.

THEOREM 1. Suppose $\Lambda_{1} \subset \Lambda_{2}, \Lambda_{1} \neq \Lambda_{2}$ and $K_{W}$ is any of the topologies on $\Lambda_{2}$ described in $\S 3$, then $\Lambda_{1}$ is of the first category in $\left(\Lambda_{2}, K_{W}\right)$.

Proof. There exists $k \geqq 0$ such that $k \in \Lambda_{1}^{*} \sim \Lambda_{2}^{*}$. The set $I=\left(h \in \Lambda_{1}^{*}:|h| \leqq k\right)$ is weakly bounded in $\Lambda_{1}^{*}$. Therefore 


$$
I^{0}=\left(f \in \Lambda_{1}:\left|\int f g d \mu\right| \leqq 1 \text { for all } g \in I\right)
$$

is an absorbing set in $\Lambda_{1}$, that is, $\Lambda_{1}=\bigcup_{n=1}^{\infty} n I^{0}$. We will show that $I^{0}$ is nowhere dense in $\left(\Lambda_{2}, K_{W}\right)=\Lambda_{2}$, completing the proof.

Suppose the closure $\tilde{I}^{0}$ of $I^{0}$ in $\Lambda_{2}$ contains an interior point. Let $U$ be a neighborhood contained in $I^{0}$. The smallest convex normal subset in $\Lambda_{2}$ containing $U$ is both contained in $I^{0}$ and is a neighborhood of 0 . Thus, $I^{0}$ is a neighborhood of 0 in $\Lambda_{2}$. This implies that $\bigcup_{n=1}^{\infty} n \bar{I}^{0}=\Lambda_{2}$, which in turn implies the existence of a function $f$ in $\bar{I}^{0}, f \geqq 0$, such that

$$
\int f k d \mu=+\infty
$$

Let $\left(f_{\alpha}\right)$ be a net in $I^{0}$ which converges $K_{W}$ to $f$. Because the net $\left(f_{\alpha} \vee 0\right) \wedge f$ is also in $I^{0}\left(I^{0}\right.$ is a normal set), we may assume that $0 \leqq f_{\alpha} \leqq f$ for every $\alpha$.

Recall, we have the sets $E_{1} \subset E_{2} \subset \cdots$ in $\mathscr{C}$ such that $\bigcup_{n=1}^{\infty} E_{n}=E$; choose $i$ such that

$$
\int E_{i} f k d \mu>1
$$

Set $E_{i}=F$ and give the space $\Lambda_{F}=\left(h \in \Lambda_{2}: \chi(F) h=h\right)$ the topology induced by $K_{W}$. The space $\Lambda_{F}$ can be regarded as a subspace of $L_{F}^{1}$. Since the injection mapping of $\Lambda_{F}$ into $L_{F}^{1}$ is continuous, the topology induced in $\Lambda_{F}$ by that of $L_{F}^{1}$ is weaker than that induced in $\Lambda_{F}$ by $K_{W}$. The result of all this is that $\left(\chi(F) f_{\alpha}\right)$ converges in the norm topology of $L_{F}^{1}$ to $\chi(F) f$. This being the case, we can extract from the net $\left(\chi(F) f_{\alpha}\right)$ a subsequence $\left(h_{i}\right)$ which converges to $f$ a.e. Now, by Fatou's lemma, we have

$$
\begin{aligned}
\lim \inf \int_{F} h_{i} k d \mu & \geqq \int_{F} \lim \inf h_{i} k d \mu \\
& =\int_{F} f k d \mu>1 .
\end{aligned}
$$

Each function $h_{i} \in I^{0}$, while $k \in I$; moreover, there exists $i_{0}$ such that

$$
\int h_{i_{0}} k d \mu>1
$$

which is a contradiction. Hence $\tilde{I}^{0}$ does not contain an interior point and is, therefore, nowhere dense in $\Lambda_{2}$.

The idea for the proof of (2) implies (3) in the next result is due to Halpern; it was used by Luxemburg in [12] to prove a related result. The idea for the proof of part b in (4) implies (1) arose in a discussion I had with Professor Calderón concerning the Banach spaces of Luxemburg. 
THEOREM 2. Give $\Lambda$ the topology $K$. The following four statements are equivalent:

(1) $\Lambda^{*}$ can be identified with the topological dual of $\Lambda$.

(2) Weakly bounded, weakly closed subsets of $\Lambda^{*}$ are weakly compact.

(3) If $\left(f_{n}\right)$ is any sequence in $\Lambda$ such that $f_{n} \downarrow 0$ then $S_{B}\left(f_{n}\right) \downarrow 0$ for every weakly bounded normal subset $B$ of $\Lambda^{*}$.

(4) $\Lambda$ is separable, i.e., contains a countable dense subset.

Proof. The implication (1) implies (2) follows from [1, p. 68] and Lemma 3.

To show (2) implies (3) let $\left(f_{n}\right)$ be a sequence in $\Lambda$ such that $f_{n} \downarrow 0$ and let $B$ be any weakly bounded normal subset of $\Lambda^{*}$. Since $S_{\bar{B}}^{-}(f) \geqq S_{B}(f)(\bar{B}$ is the weak closure of $B$ in $\Lambda^{*}$ ), we assume that $B$ is weakly closed. Suppose $\varepsilon>0$ is given and consider the sets

$$
B_{n}=\left(g \in \Lambda^{*}: \int f_{n} g d \mu<\varepsilon\right) .
$$

Because $f_{n} \downarrow 0$, we have that $\left|f_{n} g\right| \downarrow 0$ so that

$$
\int f_{n} g d \mu \leqq \int\left|f_{n} g\right| d \mu \downarrow 0
$$

for every $g \in \Lambda$. Consequently if $g$ is any element of $\Lambda^{*}$ there exists some $n$ such that $g \in B_{n}$. Each of the sets $B_{n}$ is open in the weak topology of $\Lambda^{*}$; therefore, $\left(B_{n}\right)$ is an open covering of $B$. Choose indices $n_{1}, n_{2}, \cdots, n_{k}$ such that $B \subset \bigcup_{i=1}^{k} B_{i}$.

Notice that if $g \in B$, then $|g| \in B$; so for any $g \in B$ there exists $n_{i}$ such that $1 \leqq i \leqq k$ and

$$
\int f_{n_{i}}|g| d \mu<\varepsilon
$$

Let $N=\max _{1 \leqq i \leqq k}\left(n_{i}\right)$. If $n \geqq N$, we have

$$
\int f_{n} g d \mu \leqq \int f_{n}|g| d \mu \leqq \min _{1 \leqq i \leqq k} \int f_{n_{i}}|g| d \mu<\varepsilon .
$$

Hence

$$
S_{B}\left(f_{n}\right)=\sup _{g \in B} \int f_{n} g d \mu<\varepsilon
$$

for every $n \geqq N$. The implication (2) implies (3) is complete.

Let us show that (3) implies $\Lambda$ is separable.

First, it is clear that the separability of $\Lambda$ follows from that of $\Lambda^{+}=(f \in \Lambda: f \geqq 0)$ when this subset is given the induced topology. Since every nonnegative measurable function is the monotone limit of simple functions, the proof will be complete as soon as we show that the space of nonnegative simple functions in $\Lambda$ is a separable set with the induced topology. Here is the first place 
where (3) must be used; for, suppose $f_{n}$ is a sequence of simple functions in $\Lambda^{+}$ such that $f_{n} \uparrow f$ for some $f \in \Lambda$; then $f-f_{n} \downarrow 0$ so that $S_{B}\left(f-f_{n}\right) \downarrow 0$. Lastly, because each simple function in $\Lambda^{+}$is a monotone limit of simple functions whose range is the rationals, it suffices to show that the subset $(\chi(F): \mu(F)<\infty)$ of $\Lambda^{+}$is separable when it is given the induced topology.

Our measure space is separable, so there exists a sequence $\left(F_{n}\right)$ of measurable sets such that given any measurable set $F$ and any $\varepsilon>0$ there exists $n$ such that $\mu\left(\left(F \sim F_{n}\right) \cup\left(F_{n} \sim F\right)\right)<\varepsilon$. To the sets $\left(F_{n}\right)$ can be added the sets $\left(F_{n} \cap E_{m}\right)$ without changing the countability of $\left(F_{n}\right)$ or its other property. We assume this was done at the outset.

To complete the proof of this implication, we must show that given any measurable set $F, \mu(F)<\infty$, any weakly bounded normal subset $B$ in $\Lambda^{*}$ and any $\varepsilon>0$ there exists $n$ such that $S_{B}\left(\chi(F)-\chi\left(F_{n}\right)\right)<\varepsilon$. Let us show that $\mu\left(H_{n}\right) \rightarrow 0$ implies $S_{B}\left(\chi\left(H_{n}\right)\right) \rightarrow 0$. Suppose this is not the case; that is, there exists $\eta>0$ such that $S_{B}\left(H_{n}\right)>\eta$ for infinitely many $n$. We may assume this inequality holds for all $n$. Pick $n(1)$ such that $\mu\left(H_{n(1)}\right)<1 / 2$; having picked $n(k-1)$, pick $n(k)$ such that $\mu\left(H_{n}(k)\right)<1 / 2^{k}$. Let $H_{m}^{\prime}=\bigcup_{k=m}^{\infty} H_{n(k)}$. Notice that $\chi\left(H_{n(m)}^{\prime}\right) \downarrow 0$; so that $S_{B}\left(\chi\left(H_{n(m)}^{\prime}\right)\right) \downarrow 0$. Choose $m_{0}$ such that $S_{B}\left(\chi\left(H_{m_{0}}^{\prime}\right)\right)<\eta$. Because $H_{m_{0}} \subset H_{m_{0}}^{\prime}$, the inequality $S_{B}\left(\chi\left(H_{m_{0}}\right)\right) \leqq S_{B}\left(\chi\left(H_{m_{0}}^{\prime}\right)\right)<\eta$ yields a contradiction.

Now notice $\mu\left(F \sim E_{n}\right) \rightarrow 0$, so there exists $i$ such that $S_{B}\left(\chi\left(F \sim E_{i}\right)\right)<\varepsilon / 2$. Next, within the set $E_{i}$, we can find a subsequence of $\left(F_{n}\right)$ call it $\left(F_{n}^{\prime}\right)$ such that $\mu\left(\left(F_{n}^{\prime} \sim E_{i} \cap F\right) \cup\left(E_{i} \cap F \sim F_{n}^{\prime}\right)\right) \rightarrow 0$ as $n \rightarrow \infty$. Thus, a $j$ exists such that $S_{B}\left(\chi\left(F_{j}^{\prime}\right)-\chi\left(E_{i} \sim F\right)\right)<\varepsilon / 2$. Hence

$$
S_{B}\left(\chi(F)-\chi\left(F_{j}^{\prime}\right)\right) \leqq S_{B}\left(\chi\left(F \sim E_{i}\right)\right)+S_{B}\left(\chi\left(F_{j}^{\prime}\right)-\chi\left(E \sim E_{i}\right)\right)<\varepsilon .
$$

Before turning to the implication (4) implies (1), we establish four preliminary facts.

(a) The space $\Lambda$ is a topological vector lattice; thus, the topological dual of $\Lambda$ is contained in the order dual; this merely says every continuous linear form $G$ on $\Lambda$ can be written as the difference of two positive linear forms $G^{+}$and $G^{-}[2$, p. 35]. We need slightly more here; we must have that $G^{+}$and $G^{-}$are also continuous. Even though there is no mention of topology in Bourbaki's proof of the Riesz theorem just cited, the continuity of $G^{+}$and $G^{-}$is implicit in his proof. It can be derived from his identity

$$
|G|(f)=\sup _{0 \leqq f^{\prime} \leqq f} G\left(f^{\prime}\right) .
$$

Let $U$ be a normal neighborhood of zero in $\Lambda$ on which $G$ is bounded, say by $M$. If $f \geqq 0$ and $f \in U$ then the identity implies

$$
|G|(f)=G^{+}(f)+G^{-}(f)=\sup _{0 \leqq h \leqq f} G(h) \leqq M ;
$$

which in turn implies that $G^{+}(f) \leqq M$ and $G^{-}(f) \leqq M$. 
Suppose, now that $f \in U$ is arbitrary; then

$$
\left|G^{+}\left(f^{+}-f^{-}\right)\right| \leqq\left|G^{+}\left(f^{+}\right)\right|+\left|G^{-}\left(f^{-}\right)\right|=G^{+}\left(f^{+}\right)+G^{-}\left(f^{-}\right) \leqq 2 M .
$$

Hence $G^{+}$is continuous; by a similar argument, we can show that $G^{-}$is continuous. Thus, as our first preliminary fact, we assert that every continuous linear form on $\Lambda$ is the difference of two positive continuous linear forms.

(b) Fix $E_{i}$ and let $\left(F_{n}\right)$ be a sequence of measurable sets contained in $E_{i}$ such that $\mu\left(F_{n}\right) \rightarrow 0$. Let $B$ be any weakly bounded normal subset of $\Lambda^{*}$ and $f$ any element of $\Lambda$. We show $S_{B}\left(\chi\left(F_{n}\right) f\right) \rightarrow 0$. Suppose this is not the case; we will show that this implies that $\Lambda$ is not separable, contradicting (4). Suppose $\varepsilon>0$ is such that $S_{B}\left(\chi\left(F_{n}\right) f\right) \geqq \varepsilon$ for infinitely many $n=1,2, \cdots$. Without loss of generality, we may assume this inequality is valid for all $n=1,2, \cdots$. Furthermore, by choosing a subsequence of $\left(F_{n}\right)$, if necessary, we may assume that $\sum_{n=1}^{\infty} \mu\left(F_{n}\right)<\infty$. Further yet, by considering the sets $\bigcup_{n=k}^{\infty}\left(F_{n}\right)$, again if necessary, we may assume that $F_{1} \supset F_{2} \supset \cdots$. Let $F=\bigcup_{n=1}^{\infty} F_{n}$ and let $F_{n+m}^{m}=F_{m} \sim F_{n+m}$. Notice that $\chi\left(F_{n+m}^{m}\right) f \uparrow \chi\left(F_{m}\right) f$; thus, Lemma 2 implies the existence of a number $p$ such that $C_{p}^{m}=S_{B}\left(\chi\left(F_{p}^{m}\right) f\right)>\varepsilon / 2$. Choose $p(1)$ such that $C_{p(1)}^{1}>\varepsilon / 2$; having chosen $p(k-1)$, choose $p(k)$ such that $C_{p(k)}^{p(k-1)}>\varepsilon / 2$. Let $F_{p(k)}^{p(k-1)}=H_{k}$ if $k \geqq 2$ and $F_{p(1)}^{1}=H_{1}$.

Consider the functions $h_{k}=\chi\left(H_{k}\right) f, k=1,2, \cdots ;$ they are all in $\Lambda$ and they all have disjoint supports. If $n(k)$ is a function whose domain is the natural numbers and whose range is in the two point set $(0,1)$, then $f_{n(k)}=\sum_{k=1}^{\infty} n(k) h_{k}$ is an element of $\Lambda$; for $\left|f_{n(k)}\right| \leqq|f|$. The functions $f_{n(k)}$ are uncountable in number and if $n_{1}$ and $n_{2}$ are two different sequences of 0 's and 1's , then for some $k_{0},\left|n_{1}\left(k_{0}\right)-n_{2}\left(k_{0}\right)\right|=1$ so that

$$
S_{B}\left(\sum_{k=1}^{\infty}\left[n_{1}(k)-n_{2}(k)\right] h_{k}\right) \geqq S_{B}\left(h_{k_{0}}\right)>\varepsilon / 2 .
$$

This implies that $\Lambda$ is not separable, contradicting (4).

(c) Given $\varepsilon>0, B$ any weakly bounded normal subset of $\Lambda^{*}$ and $f$ any element of $\Lambda$, then there exists $n_{0}$ such that $S_{B}\left(\left[\chi(E)-\chi\left(E_{n_{0}}\right)\right] f\right)<\varepsilon$. The proof of this fact is essentially the same as the proof of $(b)$.

(d) We use the facts from (b) and (c) to show that the simple functions whose support are contained in some of the sets $E_{n}$ are dense in $\Lambda$. We show that any $f \geqq 0$ in $\Lambda$ can be approximated by such simple functions. Let $\varepsilon>0$ and $B$ any weakly bounded normal subset of $\Lambda^{*}$ be given. By (c) there exists an $n_{0}$ such that $S_{B}\left(\left[\chi(E)-\chi\left(E_{n_{0}}\right)\right] f\right)<\varepsilon / 3$.

Let $F_{n}=\left(x \in E_{n_{0}}:|f(x)|>n\right)$. The function $\chi\left(E_{n_{0}}\right) f$ is integrable so that $\mu\left(F_{n}\right) \rightarrow 0$. Thus, by (b) there exists $n_{1}$ such that $S_{B}\left(\chi\left(F_{n_{1}}\right) f\right)<\varepsilon / 3$. Finally, defined on $H=E_{n_{0}} \sim F_{n_{1}}$ there exist simple functions uniformly close to $\chi(H) f$. Let $h$ be such a simple function satisfying $S_{B}(\chi(H) f-h)<\varepsilon / 3$. The proof of (d) is complete now, for 


$$
S_{B}(f-h) \leqq S_{B}(\chi(H) f-h)+S_{B}\left(\chi\left(F_{n_{1}}\right) f\right)+S_{B}\left(\chi\left(E-E_{n_{0}}\right) f\right)<\varepsilon .
$$

The proof of the last implication now goes along the same lines as the proof of the theorem : $L^{q}$ is the dual of $L^{p}$. Let $G$ be any continuous linear form of $\Lambda$. Because of (a), we may assume that $G$ is a positive linear form on $\Lambda$. For any measurable subset $F$ of $E_{n}$ define $v_{n}(F)=G(\chi(F))$.

It follows from the continuity of $G$ and from (b) that $v_{n}$ is absolutely continuous with respect to $\mu$. Thus, by the Radon-Nikodym theorem, there exists an integrable function $g_{n}$ such that

$$
v_{n}(F)=\int_{F} g_{n} d \mu
$$

for every measurable subset $F$ of $E_{n}$. Because of the total $\sigma$-finiteness of the measure space, there exists a measurable function $g$ such that $\chi\left(E_{n}\right) g=g_{n}$ for every $n=1,2, \cdots$. Since $\chi\left(E_{n}\right) g$ is integrable, for every $n=1,2, \cdots, g h$ is integrable and

$$
G(h)=\int g h d \mu
$$

whenever $h$ is a simple function with support contained in one of the sets $E_{n}$. Thus, the two linear forms $G(h)$ and $\int g h d \mu$ agree on a dense subset of $\Lambda$. They thave unique continuous extensions to all of $\Lambda$. The proof will be complete as soon as we show that $g \in \Lambda^{*}$. To do this, we must show $\int g f d \mu<\infty$ for every $f \in \Lambda$. It is easy to show that $g \geqq 0$; thus, we need only show $\int f g d \mu<\infty$ for $f \geqq 0$ in $\Lambda$. Let $f_{n} \geqq 0$ be a sequence of simple functions such that support of $f_{n}$ is contained in $E_{n}$ and such that $f_{n} \uparrow f$. Because $g \geqq 0$, we have $f_{n} g \uparrow f g$. $G$ is positive so $G\left(f_{n}\right) \leqq G(f)$. Hence

$$
\int f_{n} g d \mu \leqq G(f)
$$

for all $n=1,2, \cdots$. Therefore,

$$
\int f g d \mu=\lim _{n \rightarrow \infty} \int f_{n} g d \mu \leqq G(f),
$$

which shows that $g \in \Lambda^{*}$.

Let $\Phi$ and $\Psi$ be complementary Young's functions and let $L_{\Phi}$ and $L_{\Psi}$ be the associated Orlicz spaces with norms respectively \|\|$_{\Phi}$ and \|\|$_{\Psi}$. If we set $\Lambda=L_{\Phi}$, then $\Lambda^{*}=L_{\Psi}$. Furthermore, the uniformity $K$ on $L_{\Phi}$ is the same as the uniformity determined by the norm \|\|$_{\Phi}$. We have

COROLlary. The following five conditions are equivalent:

(1) There exist constants $u_{0} \geqq 0$ and $M \geqq 0$ such that $\Phi(2 u) \leqq M \Phi(u)$ for every $u \geqq u_{0}$.

(2) $L_{\Psi}$ can be identified with the dual of $L_{\Phi}$. 
(3) Weakly bounded weakly closed subsets of $L_{\Phi}$ are weakly compact.

(4) If $\left(f_{n}\right)$ is a sequence in $L_{\Phi}$ such that $f_{n} \downarrow 0$, then $\left\|f_{n}\right\|_{\Phi} \downarrow 0$.

(5) $L_{\Phi}$ is separable.

Proof. Zaanen in [13, p. 83] proves (1) implies (5) and (1) implies (2); while Lorentz in [10] proves that not (1) implies the simple functions are not dense in $L_{\Phi}$. The rest follows from Theorem 2 by noting that when we showed the separability of $\Lambda$ we showed a countable set of simple functions is dense in $\Lambda$.

\section{REFERENCES}

1. N. Bourbaki, Espaces vectoriels topologiques, Chapters III-V, Actualités Sci. Ind., Hermann, Paris, 1953.

2. - Intégration, Chapters I-IV, Actualités Sci. Ind., Hermann, Paris, 1952.

3. M. J. Cooper, Coordinated linear spaces, Proc. London Math. Soc. (3) 3 (1953), 305-327.

4. J. Dieudonné, Sur les espaces de Köthe, J. Analyse Math. 1 (1951), 81-115.

5. C. Goffman, Completeness in topological vector lattices, Amer. Math. Monthly 66(1959), 87-92.

6. P. R. Halmos, Measure theory, Van Nostrand, New York, 1950.

7. G. Köthe and O. Toeplitz, Lineare Räume mit unendlichen Koordinaten, J. Reine Angew. Math. 171 (1934), 193-226.

8. G. Köthe, Die Stufenräume, ein einfache Klasse linear vollkommener Räume, Math. Z. 51 (1948), 317-345. 70-80.

9. — Neubegründung der Theorie der vollkommenen Räume, Math. Nachr. 4 (1951),

10. G. G. Lorentz, Relations between function spaces, Proc. Amer. Math. Soc. 12 (1961), 127-132.

11. G. G. Lorentz and D. G. Wertheim, Representation of linear functionals on Köthe spaces, Canad. J. Math. 5 (1953), 568-575.

12. W. A. J. Luxemburg, Banach function spaces, Thesis, Technische Hogeschool te Delft, 1955.

13. A. Zaanen, Linear analysis, Interscience, New York, 1953.

UnIVERSITy of ChicAGo,

Chicago, Illinois 